\title{
Relationships between Sit-Ups and Abdominal Flexion Strength Tests and the Thickness of Each Abdominal Muscle
}

\author{
Takanori Noguchi ${ }^{1}$, Shinichi Demura ${ }^{2}$, Kenji Takahashi $^{3}$ \\ ${ }^{1}$ University of Technology, Fukui, Japan \\ ${ }^{2}$ Graduate School of Natural Science \& Technology, Kanazawa University, Kanazawa, Japan \\ ${ }^{3}$ Teikyo Heishei University, Ichihara, Japan \\ Email: t-noguchi@fukui-ut.ac.jp
}

Received April $1^{\text {st }}, 2013$; revised May $4^{\text {th }}, 2013$; accepted May $17^{\text {th }}, 2013$

\begin{abstract}
Copyright (C) 2013 Takanori Noguchi et al. This is an open access article distributed under the Creative Commons Attribution License, which permits unrestricted use, distribution, and reproduction in any medium, provided the original work is properly cited.
\end{abstract}

\begin{abstract}
In exercise prescription and training, a simple test that accurately evaluates abdominal strength without the use of a special device is required. This study aimed to examine the relationships between sit-ups and abdominal flexion strength tests and between both the above tests and the thickness of each abdominal muscle. Subjects comprised 13 healthy young males (age: $18.9 \pm 0.64 \mathrm{yr}$, height: $170.2 \pm 0.70 \mathrm{~cm}$, and weight: $64.9 \pm 8.80 \mathrm{~kg}$ ). Sit-ups and abdominal flexion strength tests as well as muscle thickness measurements of rectus abdominis, external oblique, internal oblique, and transversus abdominis muscles were performed. The relationships between the measured values were examined using Pearson's correlation coefficient. A significant correlation $(\mathrm{r}=0.75)$ was found between sit-ups and abdominal flexion strength tests. In addition, both tests showed significant correlations $(r>0.65)$ with thicknesses of the rectus abdominis and internal oblique muscles. From the present results, the relationship between sit-ups and abdominal flexion strength tests is very strong, and both tests are related to muscle thicknesses of rectus abdominis and internal oblique muscles. The sit-ups test can evaluate not only muscle endurance of abdomen but also static strength.
\end{abstract}

Keywords: Ultrasound Imaging; Abdominal Muscle; Muscle Thickness; Field Test

\section{Introduction}

It is important to accurately understand the effect of strength training over specific periods of time to efficiently reinforce strength (Pardis, Ramin, Saeed, \& Mohsen, 2012; Roger \& Thomas, 2010). Further, it is important to be able to easily determine muscle strength using methods such as grip strength. However, there are some regions whose muscle strength can be easily measured and those for which it is more difficult to be measured. Muscle strength is broadly divided into static and dynamic strengths, with the former being generally measured by maximal strength, such as grip strength. Determining maximum ability is essential for deciding relative loads in training or exercise prescription and should be adequately measured. The abdominal muscle groups contribute in enhancing intra-abdominal pressure, stabilizing the vertebral column, and maintaining posture (Michael, Erik, \& Udo, 2010). Further, they are related to flexion, torsion, and lateroflexion of the trunk and are important not only for athletes but also for common people. Hence, many training methods aiming towards reinforcing abdominal muscle groups have been proposed (Sands \& McNeal, 2002). However, devices that can easily measure abdominal muscle strength are less developed.

During the sit-ups test, subjects repeatedly bend and extend the torso at the hip for a specific period of time; hence, endurance of abdominal and hip muscles is largely related (Demura, 2011). The relative simplicity of the sit-ups test and not requir- ing special devices makes this test extremely practical. Therefore, the sit-ups test is also one of the physical fitness tests used by the Ministry of Education, Culture, Sports, Science and Technology of Japan. Given that maximal strength is also an important factor that determines muscle endurance, if the maximal abdominal strength related to sit-ups is superior, it is assumed that abdominal muscle endurance is also superior. Hence, we hypothesized that the relationship between sit-ups and abdominal flexion strength tests is strong.

Miyamoto et al. (2006) reported that knee extension strength is related to muscle thicknesses of rectus femoris and vastus intermedius (Miyamoto et al., 2007). As muscle thickness is also related to muscle volume (Abe, Kearns, \& Fukunaga, 2003; Akagi et al., 2009), it is inferred that if muscle thickness is large, static strength and muscle endurance involved in the related parts develop accordingly. Hence, it is assumed that strength measured by sit-ups and abdominal flexion strength tests is related to thicknesses of abdominal muscles.

This study aimed to examine relationships between sit-ups and abdominal flexion strength tests compared to each other and thicknesses of four major abdominal muscle groups.

\section{Methods}

\section{Subjects}

Subjects comprised 13 young males (age: $18.9 \pm 0.64 \mathrm{yr}$, 
height: $170.2 \pm 0.70 \mathrm{~cm}$, and weight: $64.9 \pm 8.80 \mathrm{~kg}$ ).

All subjects were healthy and physically competent. Before the experiment, the purpose and procedures were explained in detail, and written informed consent was obtained from all participants. The experimental protocol was approved by the Ethics Committee on Human Experimentation of the Faculty of Human Science, Kanazawa University (No. 2012-14).

\section{Procedures}

In this study, we measured sit-ups, abdominal flexion strength, and muscle thickness. Each measurement device and procedure was as follows.

\section{Sit-Ups Test}

The test was performed according to the recommended method for physical fitness tests by the Ministry of Education, Culture, Sports, Science and Technology. Subjects lay on their back with their knees bent at approximately right angles while both feet were positioned flat on the ground. They held their hands against their chest where they must remain throughout the test (Figure 1). During the test, an assistant held the subjects' feet placed on the ground. Subjects sat up until they touched their knees to both elbows; then, they returned to the floor. The movement was repeated as many times as possible for $30 \mathrm{~s}$. The assistant counted and records the number of correct completed sit-ups. The test was measured only once owing to the influence of fatigue.

\section{Abdominal Flexion Strength Test}

A trunk strength measurement device (original model: Takei Scientific Instruments Co. Ltd. Tokyo, Japan) was used to measure strength of abdominal flexion. This device was newly developed to measure isometric strength during forward bending of the trunk. While pushing the rotation lever at chest height, the exerted strength was measured by a strain-gauge meter connected through a pulley (Figure 2).

After adjusting the chair height to match the seated height of each subject, subjects sat so as to match their abdominal flexion

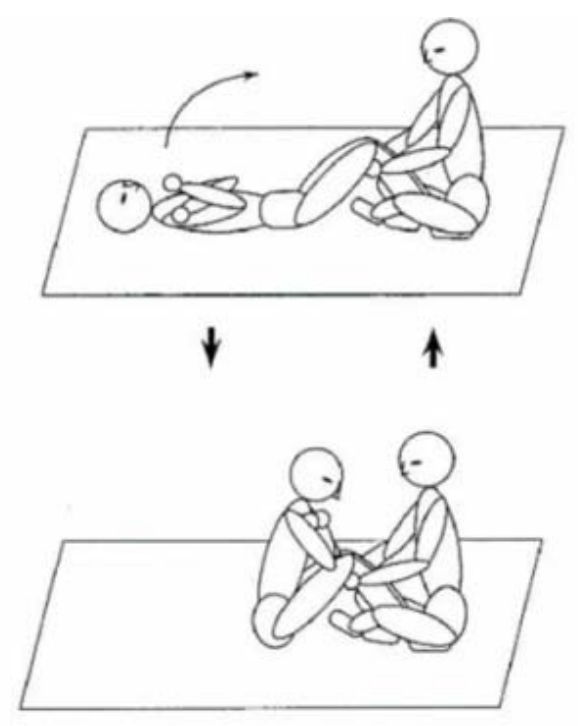

Figure 1.

Sit-ups test.

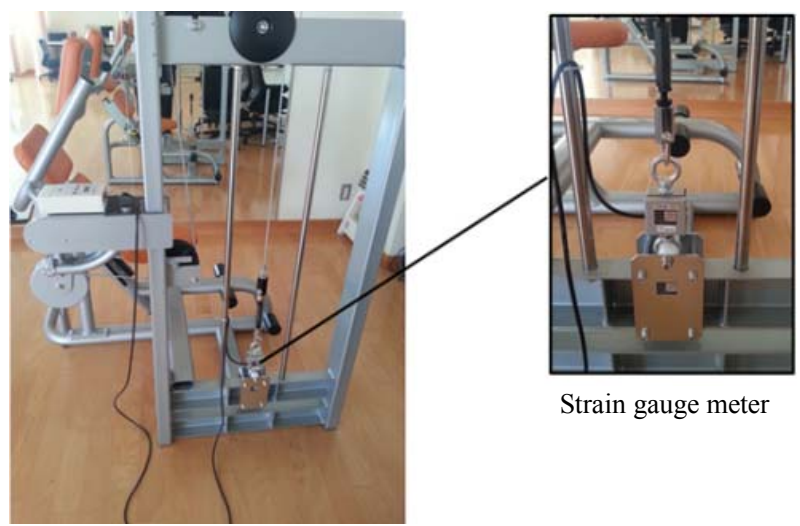

Figure 2.

Trunk strength measurement device.

point on the rotation axis of the lever. After placing both elbows on the base of the rotation lever with arms folded, subjects exerted abdominal flexion strength maximally with their timing. At that time, the tester checked that the subject did not push with their hands or back. The measured value through a pulley was revised by the following formula:

Abdominal flexion strength $=$ measurement value $(\mathrm{kg}) \times 0.6$

After a single practice, the test was performed twice. A one minute rest was taken between each trial to eliminate the influence of fatigue.

\section{Thickness of Abdominal Muscles}

An ultrasound imaging device (GT-101, TANITA, Tokyo, Japan) was used to measure thicknesses of abdominal muscles. Figure 3 shows computer displayed ultrasound images using the B-mode method. Thicknesses of four muscles (rectus abdominis, external oblique muscle, internal oblique muscle, and transversus abdominis) were measured using a probe frequency of $6 \mathrm{MHz}$. The thickness of rectus abdominis was measured as the maximal width of a point $4-\mathrm{cm}$ transverse from the navel, excluding tendinous intersections. Because the other three parts have overlapping organizations, they were measured at the same position (Ferreira, Ferreira, \& Hodges, 2004; Leonardo, Chris, Jane, Paul, \& Debra, 2009). We measured two thirds of line drawn horizontally from the height of the navel perpendicular to a vertical axillary line (Figure 4).

All measurements were completed by a single experienced tester. The thickness of each abdominal muscle was measured twice in an upright standing position with muscles tensed to increase abdominal pressure.

\section{Statistical Analyses}

Intra-class correlation coefficients (ICC) were calculated to evaluate trial-to-trial reliability. The relationships between situps and abdominal flexion strength tests that and between both tests and the thickness of abdominal muscle was examined by Pearson's correlation coefficient. Statistical significance $(\alpha)$ was set at $p<0.05$.

\section{Results}

Table 1 shows the results of ICCs of abdominal flexion strength test, and the thickness of each abdominal muscle. All 

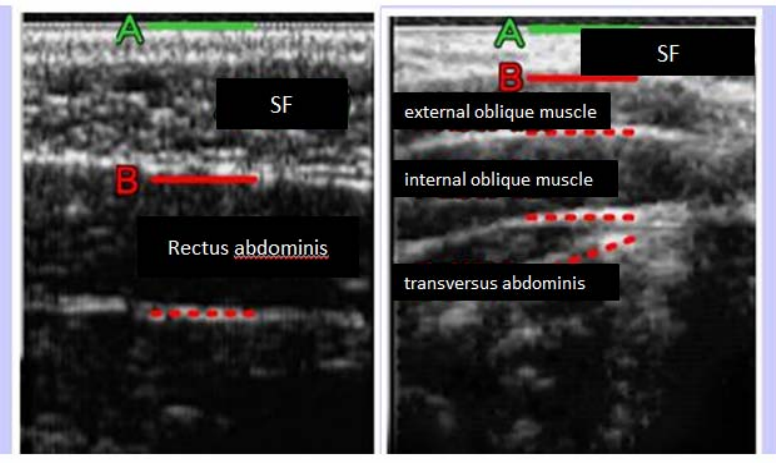

Figure 3.

Ultrasound imaging.
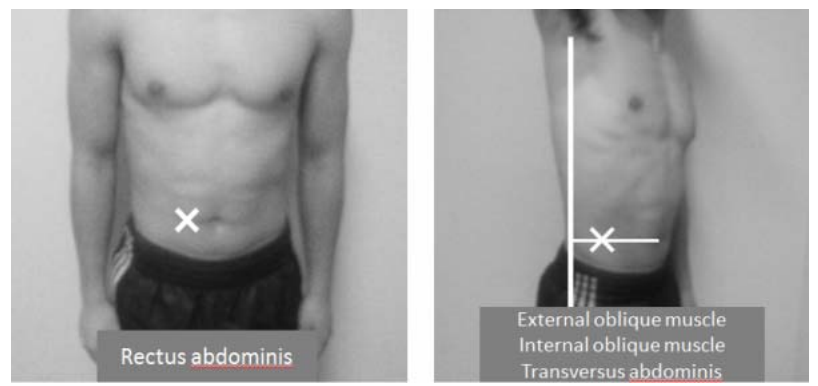

Figure 4.

Measurment sites of ultrasound imaging. variables showed a very high ICC over 0.94 . Table 2 shows the correlation between sit-ups and abdominal flexion strength tests and that between both the above tests and the thickness of each abdominal muscle. A significant and high correlation was found between sit-ups and abdominal flexion strength tests $(r=$ 0.75). Significant and moderate or higher correlations were found between both the above tests and thickness of rectus abdominis and internal oblique muscles $(r=0.65$ and 0.79 , respectively).

\section{Discussion}

In both strength testing and ultrasound imaging analyses, the reliability of measured values is often questioned (Hides, Miokovic, Belavy, Stanton, \& Richardson, 2007; Mannion et al., 2008). ICC of abdominal flexion strength in this study was very high (0.99). This indicates that the values measured by the trunk strength measurement device, developed in this study, have high reliability. In addition, ICCs of the thickness of each abdominal muscle was also very high, with all values over 0.94 . Gill, Mason, \& Gerber (2012) reported that ICCs of the thickness of transverse abdominal muscle, external oblique muscle, and internal oblique muscle measured by the same method were approximately $0.97-0.98$. On the basis of results, the reliability of muscle thicknesses of individual abdominal muscles as measured by ultrasound imaging is considered to be high.

Measurement of abdominal flexion strength is impractical as a field test in actual training because it requires a specialized device (Jungmin, Hyoseob, Se, \& Yu, 2012). In contrast, the

Table 1.

Intra-class correlation coefficients (ICCs) of abdominal flexion strength, and each part of abdominal muscle thickness.

\begin{tabular}{lcccccccc}
\hline & \multicolumn{3}{c}{ 1st } & \multicolumn{3}{c}{ 2nd } & & \\
\hline & & Mean & SD & Mean & SD & F-value & $p$ & ICC \\
Strength & $(\mathrm{kg})$ & 46.7 & 17.84 & 46.7 & 17.76 & 0.02 & 0.90 & 0.99 \\
Rectus abdominis & $(\mathrm{mm})$ & 17.7 & 3.85 & 17.8 & 3.98 & 0.06 & 0.81 & 0.98 \\
External oblique muscle & $(\mathrm{mm})$ & 8.1 & 2.23 & 8.2 & 1.93 & 1.00 & 0.34 & 0.96 \\
Internal oblique muscle & $(\mathrm{mm})$ & 15.6 & 3.69 & 15.6 & 3.56 & 0.04 & 0.84 & 0.97 \\
Transversus abdominis & $(\mathrm{mm})$ & 5.2 & 1.19 & 5.2 & 1.53 & 0.00 & 1.00 & 0.94 \\
\hline
\end{tabular}

Note: ${ }^{*} p<0.05$.

Table 2.

Correlations between the sit-ups, abdominal flexion strength, and muscle thickness.

\begin{tabular}{|c|c|c|c|c|c|c|c|}
\hline \multirow[b]{2}{*}{$\mathrm{n}=13$} & & \multirow[b]{2}{*}{ Mean } & \multirow[b]{2}{*}{ SD } & \multicolumn{4}{|c|}{ Coefficient of correlation (r) } \\
\hline & & & & The sit-ups & $p$ & Strength & $p$ \\
\hline Sit-ups & (Times) & 30.6 & 7.42 & & & & \\
\hline Strength & $(\mathrm{kg})$ & 46.7 & 17.75 & $0.75^{*}$ & 0.00 & & \\
\hline Rectus abdominis & $(\mathrm{mm})$ & 17.8 & 3.88 & $0.66^{*}$ & 0.01 & $0.65^{*}$ & 0.02 \\
\hline External oblique muscle & $(\mathrm{mm})$ & 8.1 & 2.04 & -0.15 & 0.63 & -0.02 & 0.94 \\
\hline Internal oblique muscle & $(\mathrm{mm})$ & 15.6 & 3.56 & $0.79^{*}$ & 0.00 & $0.69^{*}$ & 0.01 \\
\hline Transversus abdominis & $(\mathrm{mm})$ & 5.2 & 1.33 & -0.02 & 0.95 & 0.08 & 0.81 \\
\hline
\end{tabular}

Note: ${ }^{*} p<0.05$. strength: abdominal flexion strength. 
sit-ups test, consisting simply of repetitive abdominal flexion and extension can be easily performed anywhere and requires no equipment. The sit-ups test has been used for evaluating muscle endurance from completed sit-ups within a predetermined time or the time until completion of a predetermined number of sit-ups (American college of sports medicine, 2000). However, it is considered that static strength in addition to muscle endurance of the abdomen are related to successful completion of the sit-ups test because a high correlation was found between sit-ups and abdominal flexion strength tests in this study. In brief, it is inferred that individuals with superior abdominal flexion strength can perform and maintain rapid abdominal flexion throughout the sit-ups test.

It was reported that the magnitude of strength of a muscle group is strongly related to its thickness (Miyamoto et al., 2007). Hence, it is inferred that larger muscle thickness is also related to well-developed static strength and muscle endurance involved in strength exertion of the related parts. The abdominal muscle groups consist of the following four parts: rectus abdominis, external oblique muscles, internal oblique muscles, and transversus abdominis (Michael, Erik, \& Udo, 2010). By examining thicknesses of abdominal muscle groups related to sit-ups and abdominal flexion strength, the muscle parts that contribute to strength exertion of the abdomen can be identified. Significant correlations were found between the sit-ups test and thicknesses of rectus abdominis and internal oblique muscles ( $\mathrm{r}$ $=0.66$ and 0.79 , respectively). Rectus abdominis is a paired muscle that runs vertically on the anterior abdominal wall and is related to abdominal flexion. In additional, internal oblique muscles are located in lateral abdominal regions and are also related to abdominal flexion of rectus abdominis. It is considered that people who perform the sit-up exercise develop the above muscle groups related to abdominal flexion. Miyamoto et al. (2007) reported that knee extension strength showed significant relationship with thicknesses of rectus femoris and vastus intermedius muscles (rectus femoris muscle: $\mathrm{r}=0.56$, vastus intermedius muscle: $r=0.43$, and their total: $r=0.62$ ) (Miyamoto et al., 2007). In brief, it is suggested that people with greater muscle thicknesses are superior in strength in the corresponding parts. In the present results, significant correlations were found between the abdominal flexion strength and thicknesses of rectus abdominis and internal oblique muscles ( $\mathrm{r}$ $=0.65$ and 0.69 , respectively). Hence, abdominal flexion strength of people with well-developed rectus abdominis and internal oblique muscles may also be superior.

In contrast, the present results show that thickness of external oblique and transversus abdominis muscles showed non-significant relationships with sit-ups and abdominal flexion strength tests. While these muscle groups are anatomically related to body trunk flexion, they are perhaps more important for lateral bending and rotation of the trunk or increasing abdominal pressure. Sit-ups and abdominal flexion strength tests used in this study may not adequately reflect characteristics of these muscle groups. For these muscles, movements more specific to the muscle action will be examined.

\section{Conclusion}

The relationship between sit-ups and abdominal flexion strength tests is strong. Further, both tests are related to thicknesses of rectus abdominis and internal oblique muscles. Static strength in addition to abdominal muscle endurance contributes considerably to completion of the sit-ups test. The sit-ups test is easily executed for the evaluation of static strength as well as muscle endurance of the abdomen. It is recommended that effectiveness of abdominal strength be evaluated on the basis of the results of the sit-ups test.

\section{Acknowledgements}

This study was supported by the Japanese Society for the Promotion of Science KAKENHI Grant-in-Aid for Young Scientists (B) Number 24700673. The authors would like to thank Enago (www.enago.jp) for the English language review. The results of the present study do not constitute endorsement by Journal of Strength \& Conditioning Research.

\section{REFERENCES}

Abe, T., Kearns, C. F., \& Fukunaga, T. (2003). Sex differences in whole body skeletal muscle mass measured by magnetic resonance imaging and its distribution in young Japanese adults. British Journal of Sports Medicine, 37, 436-440. doi:10.1136/bjsm.37.5.436

Akagi, R., Takai, Y., Kato, E., Fukuda, M., Wakahara, T., Ohta, M., Kanehisa, H., Kawakami, Y., \& Fukunaga, T. (2009). Relationships between muscle strength and indices of muscle cross-sectional area determined during maximal voluntary contraction in middle-aged and elderly individuals. The Journal of Strength \& Conditioning Research, 23, 1258-1262. doi:10.1519/JSC.0b013e3181918a9b

American College of Sports Medicine (2000). ACSM's guidelines for exercise testing and prescription (6th ed.). Philadelphia, PA: Lippincott Williams \& Wilkins.

Demura, S. (2011). Health and a sports science lecture (2nd ed., pp. 105-125). Tokyo: Kyorinsyoin.

Ferreira, P. H., Ferreira, M. L., \& Hodges, P. W. (2004). Changes in recruitment of the abdominal muscles in people with low back pain: Ultrasound measurement of muscle activity. Spine, 29, 2560-2566. doi:10.1097/01.brs.0000144410.89182.f9

Gill, N. W., Mason, B. E., \& Gerber, J. P. (2012). Lateral abdominal muscle symmetry in collegiate single-sided rowers. International Journal of Sports Physical Therapy, 7, 13-19.

Hides, J. A., Miokovic, T., Belavy, D. L., Stanton, W. R., \& Richardson, C. A. (2007). Ultrasound imaging assessment of abdominal muscle function during drawing-in of the abdominal wall: An intrarater reliability study. Journal of Orthopaedic \& Sports Physical Therapy, 37, 480-486.

Jungmin, K., Hyoseob, L., Se, I. L., \& Yu, J. K. (2012). Thickness of rectus abdominis muscle and abdominal subcutaneous fat tissue in adult women: Correlation with age, pregnancy, laparotomy, and body mass index. Archives of Plastic Surgery, 39, 528-533. doi:10.5999/aps.2012.39.5.528

Leonardo, O. P. C., Chris, G. M., Jane, L., Paul, W. H., \& Debra, S. (2009). An investigation of the reproducibility of ultrasound measures of abdominal muscle activation in patients with chronic nonspecific low back pain. European Spine Journal, 18, 1059-1065. doi:10.1007/s00586-009-1018-5

Mannion, A. F., Pulkovski, N., Gubler, D., Gorelick, M., O’Riordan, D., Loupas, T., Schenk, P., Gerber, H., \& Sprott, H. (2008). Muscle thickness changes during abdominal hollowing: An assessment of between-day measurement error in controls and patients with chronic low back pain. European Spine Journal, 17, 494-501. doi:10.1007/s00586-008-0589-X

Michael, S., Erik, S., \& Udo, S. (2010). Prometheus. In Lernatlas der anatomie (pp. 130-141). Tokyo: Igakusyoin.

Miyamoto, K., Tanaka, S., Tanaka, A., Matsumura, S., Tanisawa, T., Moriyama, S., Nakatani, T., \& Tanaka, S. (2007). Appropriation of colorized ultrasonographics, and relationships between muscle thickness and muscle volume or strength. Structure and Function, 6, 27-32.

Pardis, N., Ramin, K., Saeed, D., \& Mohsen, R. (2012). The effect of 


\section{T. NOGUCHI ET AL.}

abdominal resistance training and energy restricted diet on lateral abdominal muscles thickness of overweight and obese women. Journal of Bodywork and Movement Therapies, 16, 344-350.

doi:10.1016/j.jbmt.2011.12.001

Sands, W. A., \& McNeal, J. R. (2002). A kinematic comparison of four abdominal training devices and a traditional abdominal crunch. The Journal of Strength and Conditioning Research, 16, 135-141.

Roger, W. E., \& Thomas, R. B. (2010). NSCA's essentials of personal training (pp. 49-78). Tokyo: MORINAGA \& CO., LTD. 